\title{
Novel Visual Perceptual Phenomenon in Isoluminant Bi- Coloured Square-Wave Gratings
}

\author{
by \\ Azaac Tan Wei En \\ Supervisor: Asst. Prof. Gerrit Maus \\ Psychology \\ School of Social Sciences \\ College of Humanities, Arts, \& Social Sciences
}

Submitted to the School of Biological Sciences in partial fulfilment of the requirements for Final Year Project

NANYANG TECHNOLOGICAL UNIVERSITY

April, 2019 


\section{TABLE OF CONTENTS}

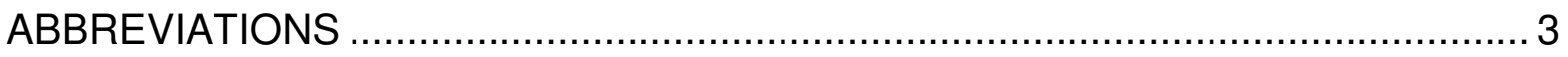

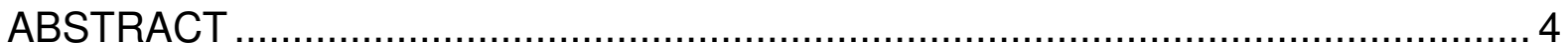

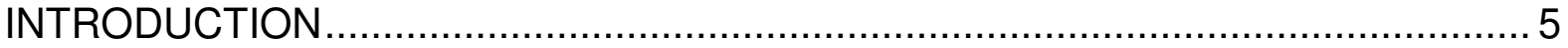

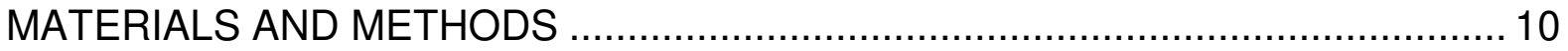

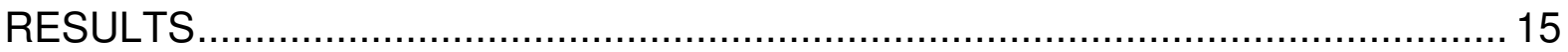

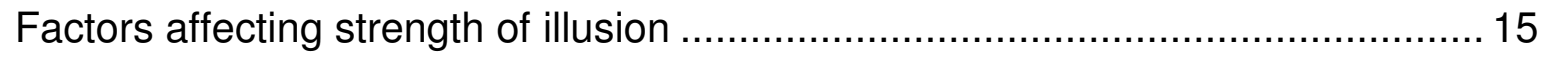

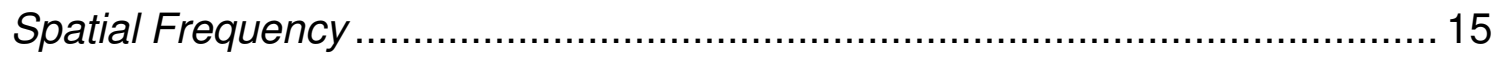

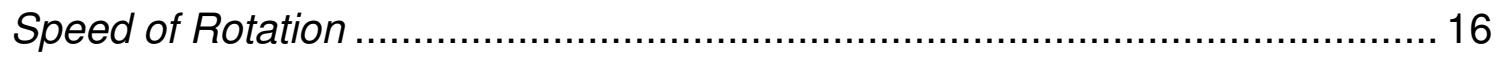

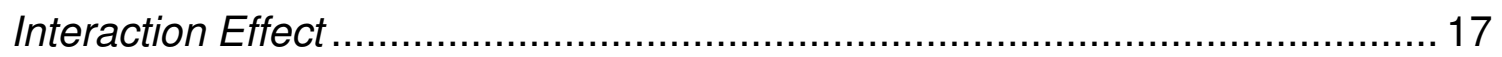

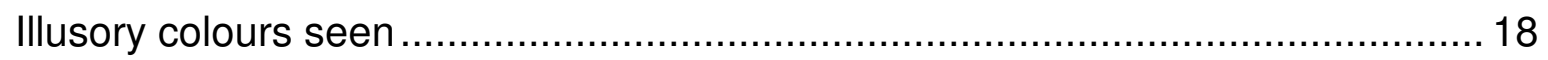

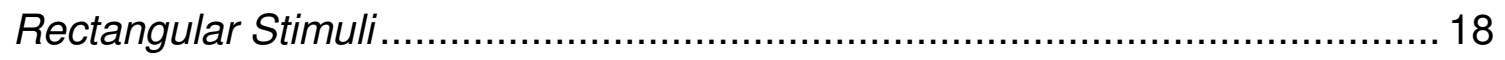

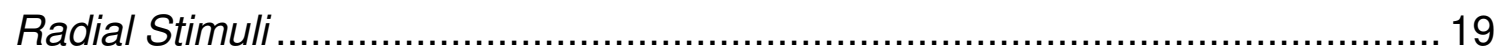

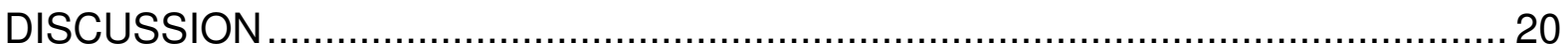

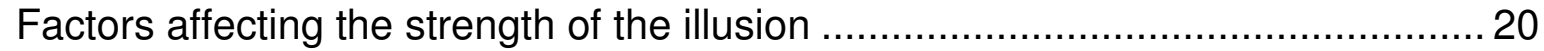

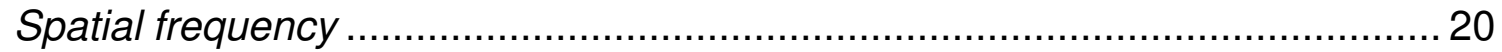

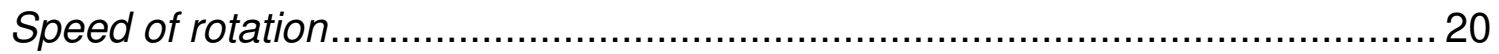

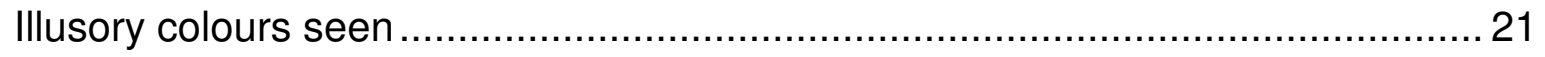

Non-Complementary Colour Pairs ....................................................... 21

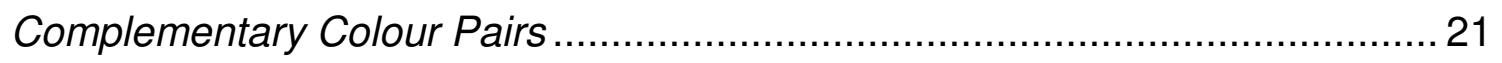

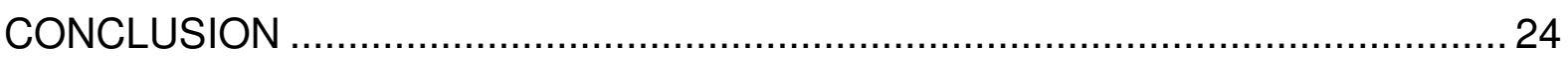

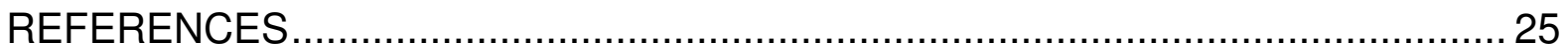




\section{ABBREVIATIONS}

\begin{tabular}{|c|c|}
\hline Abbreviated form & Extended Form \\
\hline ANOVA & Analysis of Variance \\
\hline CRT & Cathode Ray Tube \\
\hline HSV & Hue, Saturation, Value \\
\hline LGN & Lateral Geniculate Nucleus \\
\hline RGB & Red, Green, Blue \\
\hline
\end{tabular}


Novel Visual Perceptual Phenomenon in Isoluminant Bi-Coloured Square-Wave Gratings

(Azaac Tan Wei En)

\section{ABSTRACT}

Visual illusions, beyond their ability to entertain, reveal the "flaws" and limits of human perception, and how our visual senses can be fooled. In this study, we present a novel illusory percept generated by isoluminant bi-coloured square-wave gratings. We document the physical characteristics of the stimuli that affect the strength of the illusion and the illusory colours perceived, and consider potential mechanisms underlying this phenomenon. In line with our hypotheses, the results reveal that the strength of the illusion varies with the spatial frequency of the grating, and that the illusory percept is motion-dependent. Analysis of the illusory colours perceived reveals a relationship between the stimulus colours and the illusory colours perceived. This relationship demonstrates integration of the typically independent red-green parvocellular and blue-yellow koniocellular channels in the visual pathway. Overall, this pioneering study points towards a new mechanism of colour perception and integration, possibly in the early visual cortex. Possible future directions of research employing these coloured square-wave gratings are discussed. 


\section{INTRODUCTION}

\section{Colour Vision Theory}

Since the $19^{\text {th }}$ century, the field of colour vision has been primarily dominated by two theories - namely, the Young-Helmholtz trichromatic theory (1852), and subsequently the opponent-process theory by Ewald Hering (1892). Rather than being in opposition to one another, both theories are implicated in accounting for colour vision in humans, with models put forth to demonstrate the mechanism of integrating the two (Boynton, 1986).

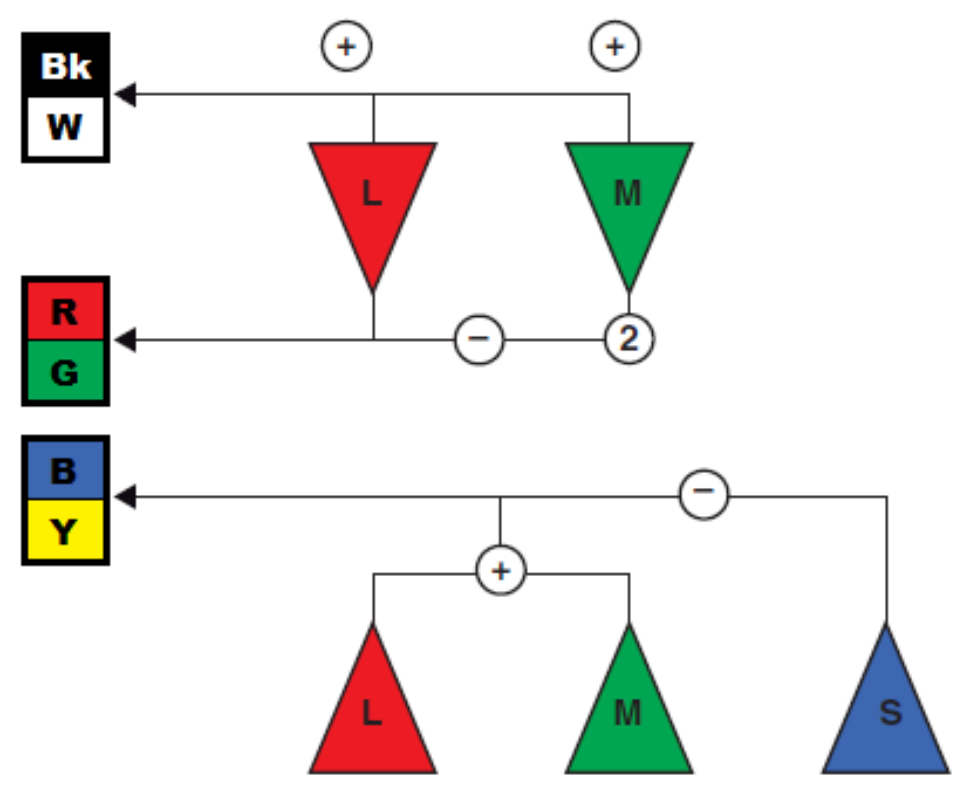

Figure 1. Integration of trichromatic and opponent-process theory in the Boynton model. Adapted from Ekroll, 2005.

Trichromatic theory works at the level of the three photoreceptors in the retina which are known as the L, M and S-cones. Each type of cone is sensitive to electromagnetic radiation of a particular range of wavelengths; long, medium and short wavelengths, respectively, collectively encompassing the range commonly referred to as the "visible spectrum" of light $(400-700 \mathrm{~nm})$. The peak sensitivities of the cones are close to wavelengths that correspond to red, green and blue, thus leading to them being known as the three "basic" colours of light.

In Hering's colour-opponent theory, he proposed four basic hue impressions: red, green, blue and yellow, of which the red-green and blue-yellow pairs were opposing colours that cannot be mixed (e.g. no reddish-green or blueish-yellow exists). To explain luminance perception, Hering further added a third opponent black-white pair. In support of Hering's theory, numerous studies have found groups of neurons which are spectrally opponent - stimulated by one of the colours in the pair and inhibited by the other. These spectrally opponent neurons, or opponent-colour cells, 
extend from early in the visual pathway, in the retina (Svaetichin \& MacNichol Jr, 1958), to the lateral geniculate nucleus (LGN) (De Valois et al., 1958; Wiesel \& Hubel, 1966), and in the visual cortex (Zeki, 1983).

\section{Refining Colour Theory}

Since the time of Young, Helmholtz and Hering, numerous phenomena pertaining to hue perception have been discovered, challenging the sufficiency of the foundational work. In 1873, Bezold documented the influence of the luminance levels of monochromatic lights on the colours perceived, leading to work revealing the nonlinear nature of signal transduction in the opponent colour system (Ejima \& Takahashi, 1984). In 1909, Abney documented the shift in hue perception caused by the addition of white light to monochromatic colours.

Furthermore, in the last decade, new work has emerged to challenge the place of colour opponency in serving as one of the fundamental theories of colour vision. In a review published in 2013, Ralph Pridmore challenged the two longstanding pairs of red-green and blue-yellow opponency, presenting evidence of three different opponent pairs: red-cyan, blue-yellow and green-magenta. In place of the concept of opponent colours, Pridmore argues that colour vision is governed by cells sensitive to complementary colour pairs.

When considering the role of colour vision in day-to-day living, one might fail to see its importance, and hence, its worth, of being studied. From an evolutionary perspective, colour vision facilitated survival by increasing efficiency in foraging for fruit and decoding fertility cues in mating, roles which have not lost their importance to date. Beyond the conscious perception of the colour of objects in the environment, studies have revealed the role of colour vision in the human visual system as a whole. Troscianko et al. (1991) showed that even in the absence of luminance differences, colour information facilitates the monocular perception of depth. Work by Lu et al. (1999) highlighted the role of colour perception as a cue for motion perception.

The role of colour perception to the human visual system has become increasingly apparent, highlighting the parallel increase in need to understand its underlying mechanisms, and whether these mechanisms are reliable. One particular field of psychophysics can serve to address both these needs - the field of visual illusions. 


\section{Visual Illusions}

Visual illusions, beyond their ability to entertain, reveal the "flaws" and limits of human perception, and how our visual senses can be fooled. As new illusions of motion, depth, luminance and colour emerge, the study of the mechanisms that cause the illusion to work can reveal new facets of, and drive the refining of the understanding of the human visual system.

While trichromatic theory and the concept of spectral opponency (or complementarity) give us a basis to predict how light beams of specific wavelengths might appear, they are unable to facilitate the prediction of the appearance of any given visual stimulus (Ekroll, 2005). That is to say, the perceived colour of any given patch or pattern is heavily influenced by the spatial and temporal context of its presentation.

When two stimuli are presented in succession on the same location on the visual field, the appearance of the second stimulus is affected by the colour and luminance of the first stimulus - a phenomenon known as successive colour contrast. In 1905, von Kries released a mathematical model to account for successive colour contrast. The model proposed that prior stimulation by the first stimulus results in temporary downward shifts in sensitivity in the L, M and S-cones in the retina, resulting in the altered hue perception when viewing the second stimulus.

When differently coloured stimuli are presented simultaneously, the colour (Walraven, 1976) and brightness (Whittle \& Challands, 1969) of a target coloured patch has been shown to be modified when the target is embedded in patches of a different colour. Collectively, the studies on simultaneous colour contrast pointed towards the existence of simultaneous contrast-coding, in which the cone excitation values of the target relative to that of the background governs the colour perceived.

\section{Similar Illusions}

In 1975, Tynan and Sekuler documented the perception of "visual phantoms", which are counter-phase illusory gratings perceived when a moving grating is obscured by a perpendicular occluder. Genter and Weisstein (1981) demonstrated that flickering, rather than motion of the grating, was sufficient to generate the phantom perception. Later, the percept was found to be dependent on neither motion nor flickering (Gyoba, 1983). Studies on this work were limited to the achromatic channel, and survived dichoptic presentation (McCourt, 1994). 


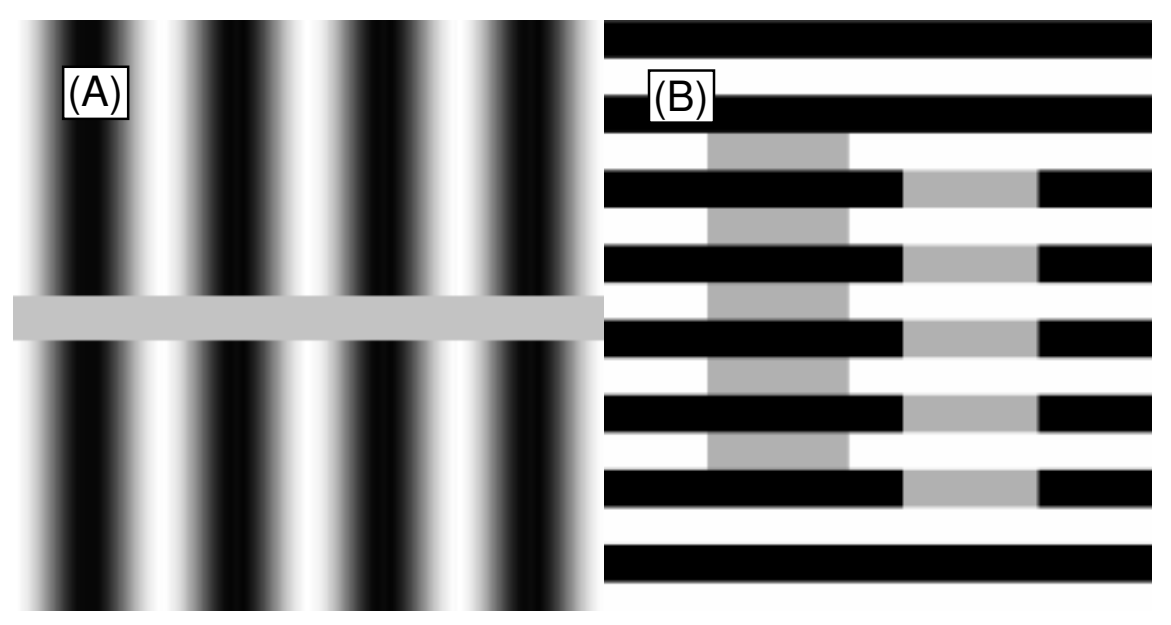

Figure 2. Illusions involving achromatic gratings (A) Phantom grating effect - a uniform grey strip perpendicular to a sine-wave grating appears to have a counter-phase luminance (B) White illusion isoluminant grey strips placed parallel to a square-wave grating appear to have different brightness

Another illusion involving achromatic grating patterns is the White illusion (White, 1979), in which an isochrominant, bi-luminant grating induces a brightness shift on a stripe of a third luminance level. In their work on the illusion, Foley and Mccourt (1985) suggested the role of end-stopped "hypercomplex" cortical cells in the perception of the illusion, while Blakeslee and McCourt (1999) implicated early-stage cortical modelling.

Earlier in 1970, Munker documented a coloured variation of the White illusion; an isoluminant, bi-colour grating induces a colour shift on a stripe of a third colour. It was later established that angular $\mathrm{T}$ - and $\mathrm{X}$-junctions are not necessary for the White and Munker illusions to work (Howe, 2005). In his study, Howe revisited both illusions, presenting radial (circular) versions which maintained the illusory perception. It is worthy to note that none of these illusions were found to be dependent on motion.

\section{Aims and Objectives}

In this study we present a novel visual illusory percept - short-lived illusory colours perceived when looking at isoluminant bi-colour square-wave gratings. For example, looking at alternating stripes of blue and yellow elicits short-lived perceptions of green and red pulses. While previous works studied the effects of gratings on an occluding stripe of a different colour or luminance, this pioneering study presents a novel illusion, and studies the percept(s) elicited solely by coloured gratings.

In an exploratory effort, this project attempts to study the effects of varying spatial frequency and motion on the strength of the illusion, while documenting the illusory colours perceived by observers in response to the colours of the stimuli. By doing so, 
we initiate the process of understanding the mechanisms responsible for the perception of the illusory colours, laying groundwork for future studies on the illusion.

Motion of the stimulus on the retina exposes the retina to an alternation of the two colours constituting the stimulus. By altering the spatial frequency of the stimulus, we vary the rate of alternation of the colours on the retina for any given retinal motion. Therefore, we predict that the number of the illusory colours perceived will vary with changes in the spatial frequency of the stimulus.

In early representations of the illusion, which were printouts, it was found that fixating at any location on the stimulus extinguished the illusory percept. Thus, we posit that the illusory percept is motion-dependent and can be elicited by presenting the stimulus in motion, with the frequency of the illusory percept being elicited varying with the speed of rotation during presentation.

Based on previous observations by the author and the team which discovered the illusory stimulus, we seek to characterise the relationship between the stimuli colours displayed and the illusory colours perceived. We predict that the illusory colours perceived are of a hue intermediate between the two stimuli colours, and the hue complementary to the first. 


\section{MATERIALS AND METHODS}

\section{Participants}

A total of 23 participants took part in this study. One third of the participants were male $(n=7)$ and the rest were female. Participants were tested with the Dvorine Pseudo-Isochromatic Plates (Dvorine, 1953) and verified to have no deficiencies in colour vision.

The experimental design was approved by the Nanyang Technological University's (NTU) Psychology Division Ethics Committee, and each participant gave written informed consent prior to the start of the experiment. Participants were recruited through the NTU Psychology Research Pool, and completed the experiment in exchange for course credit. No deception was employed during the experiment.

\section{Apparatus}

The stimuli were displayed on a 21" Cathode Ray Tube (CRT) monitor manufactured by Sun Microsystems, with a screen size of $40 \times 30 \mathrm{~cm}$. The CRT screen resolution was $1152 \times 864$ pixels, running at the refresh rate of $100 \mathrm{~Hz}$. Participants viewed the images from a chin rest placed $65 \mathrm{~cm}$ from the monitor, resulting in a stimulus field $34.2^{\circ}$ in width and $26^{\circ}$ in height. Gamma calibration for the monitor was conducted using a Konica Minolta LS110 spectrophotometer, in conjunction with the monitor calibration algorithm in PsychoPy (Peirce et al., 2019).

\section{Stimuli}

The experimental stimuli were programmed in Python 2.7.16 with the assistance of Aaron Ang, using the PsychoPy v3.0.4 (Peirce et al., 2019) package. The stimuli are square-wave gratings, each comprising two colours, with the colours alternating between adjacent bands (Figure 3). As shown in Figure 3, two patterns employing the same square-wave grating are used - one rectangular, and one radial (circular), with the latter drawing inspiration from Howe's (2005) radial version of the White illusion.

The rectangular square-wave grating had a fixed size of $1600 \times 1600$ pixels, with the number of coloured line pairs ranging from 25 to 105 (60 to 252 cycles $/ \mathrm{m}$ ) and rotation speed of 10 or 80 degrees per second. The radial square-wave grating had a fixed diameter of 1800 pixels, with the number of coloured line pairs ranging from 48 to 64 (204.8 to 273.1 cycles/m), and the stimulus being displayed statically. 

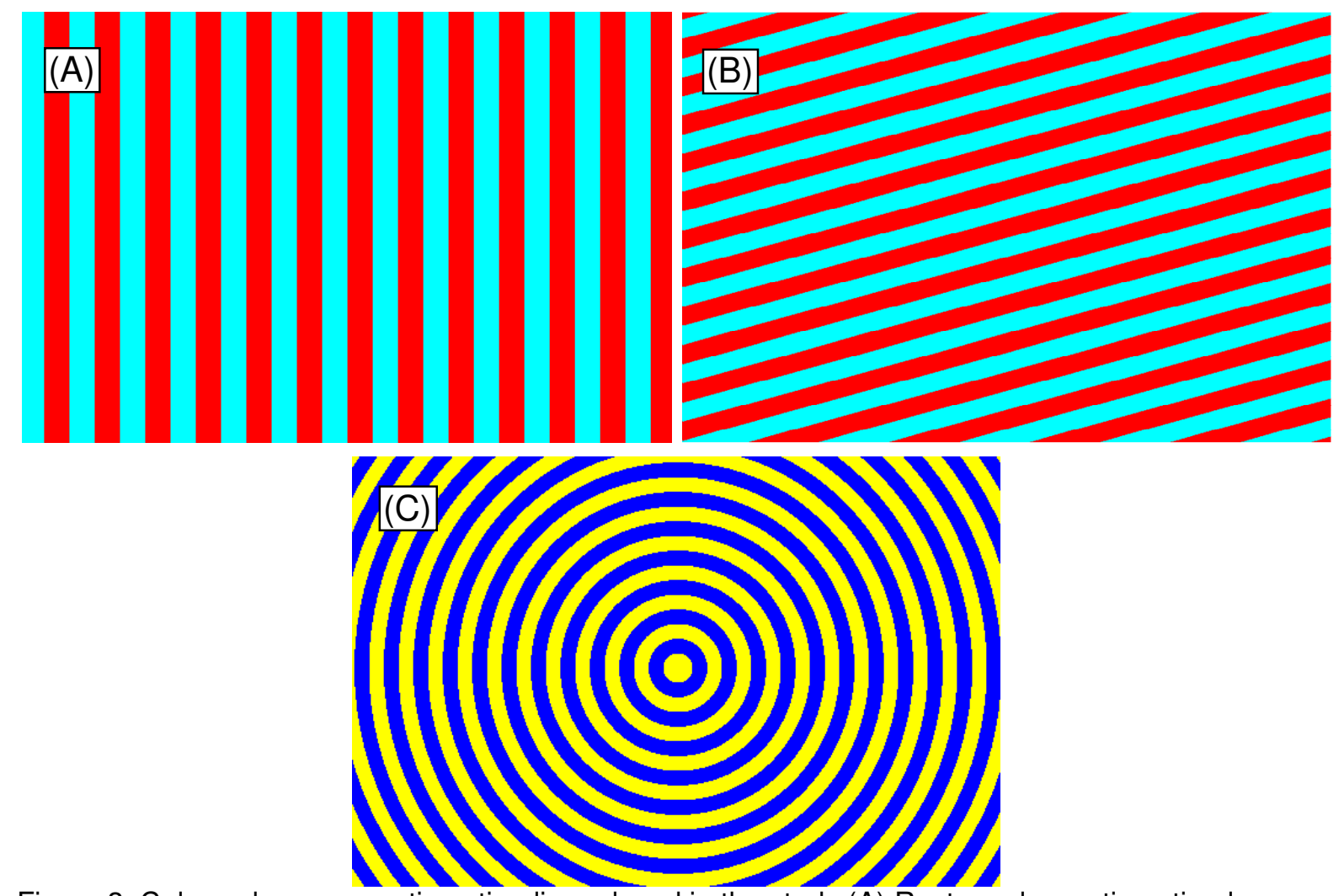

Figure 3. Coloured square-grating stimuli employed in the study (A) Rectangular grating stimulus, starting position (B) Rectangular grating stimulus, midway through rotation (C) Radial grating stimulus

While pilot tests revealed that the illusory effect of interest is maintained even when the two colours are of different luminance, work by Walraven (1976) demonstrated the effect of luminance contrasts on colour perception. Thus, efforts were made to display all stimuli at equal luminance levels. Using a spectrophotometer to compare the three (red, green and blue) phosphors in the CRT, it was determined that the maximum intensity of the blue phosphor was the lowest, at $10 \mathrm{~cd} / \mathrm{m}^{2}$. Thus, all stimuli generated were displayed at an equal luminance of $10 \mathrm{~cd} / \mathrm{m}^{2}$, with the non-primary colours yellow, magenta and cyan generated by having each constituent electron phosphor fluorescing at half the required intensity $\left(5 \mathrm{~cd} / \mathrm{m}^{2}\right.$ per phosphor).

\section{Experimental Procedure}

Participants' colour vision was tested using the Dvorine Pseudo-Isochromatic Plates (Dvorine, 1953). Participants were shown 14 plates, each containing a coloured number on a differently-coloured background. They were then tasked to identify the number(s) shown. In accordance to the analysis protocol, incorrect responses for 2 plates or fewer were considered to be due to participant's figure-ground confusion and reflective of normal colour vision. 
Participants were then shown printouts of the rectangular square-grating stimulus, and asked to identify any illusory colours seen. Once participants demonstrated that they had understood the illusion, they began a practice set on the computer workstation.

Participants were sat in front of the computer, resting their head on a chin-rest $65 \mathrm{~cm}$ away from the monitor. The practice set employed the rectangular stimuli in 6 colour combinations, and had a work flow similar to the experimental set (Figure 4), except that the stimuli were displayed for twice as long (30s) during the practice.

For each trial, after viewing a fixation cross for $1 \mathrm{~s}$, the stimulus (rectangular or radial) was presented for 15s. Participants were asked how many illusory colours they saw, and then asked to identify these illusory colours by clicking on the corresponding colours presented on a colour wheel. Lastly, participants were asked to respond on a 5-point Likert Scale how strong the illusory colours appeared.

$1 \mathrm{~s}$

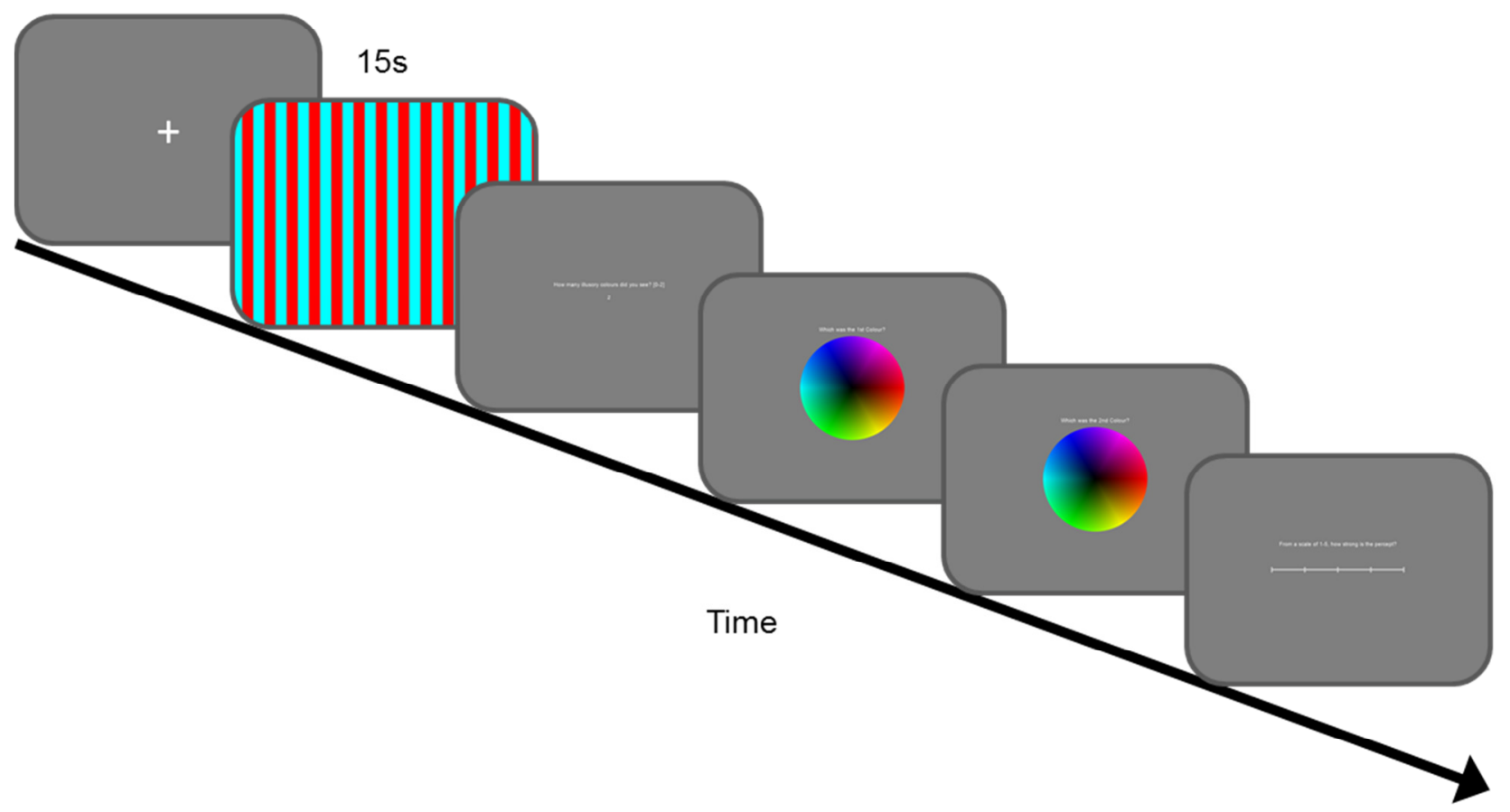

Figure 4. Flow of the experiment, for both rectangular and radial stimuli sets

After familiarisation with the flow of the experiment, participants completed the rectangular stimuli sets followed by the radial stimuli set. For the rectangular stimuli, each block of trials contained 40 trials (4 colour combinations $\times 2$ rotation speeds $\times 5$ spatial frequencies). For the radial stimuli, the block of trials contained 45 trials (15 
colour combinations $\times 3$ spatial frequencies). Participants were instructed to take eye breaks between each block of trials, and also during the blocks as they deemed necessary. In total, participants completed 3 blocks of the rectangular stimuli set (total 120 trials) and 1 block of the radial stimuli set (45 trials). Each participant took approximately 75 minutes to complete the experiment.

\section{Data Analysis}

Analysis of the participants' response data was done using IBM SPSS Statistics, Version 23.0, and Python.

\section{Illusion Strength}

For analysis of the strength of the illusion, we took the number of illusory colours seen when viewing the stimuli as a proxy. For each of the conditions (5 spatial frequencies $x 2$ rotation speeds), the illusion strength was averaged for each condition using SPSS, and graphs were generated to facilitate comparison.

As there were two independent variables (spatial frequency and speed of rotation) acting on one dependent variable (strength of illusion), a two-way repeated measures analysis of variance (ANOVA) was most appropriate for analysis. We conducted a two-way repeated measures ANOVA using SPSS, which allowed us to examine the effects of each independent variable on the illusion strength, and also to determine if there were any interaction effects between the two.

\section{Illusion Colour}

According to trichromatic theory, any visible colour can be produced by a combination of red, green and blue lights. Thus, one of the commonly employed methods of displaying colours is the Red, Green, Blue (RGB) colour space - an additive three-dimensional space.

In order to represent participants' responses on the illusory colours seen for each stimulus, we selected the Hue, Saturation, Value (HSV) representation of the Red, Green, Blue (RGB) colour space. Analysis using the three Red, Green and Blue values would require analysis to be done in three-dimensions. In contrast, the hue value is a single variable ranging from 0 to 360 degrees, facilitating simpler analysis of participants' colour responses along one dimension.

Participants' colour responses were converted from their RGB values to the corresponding HSV values using a Python script programmed with the assistance of 
Tan Quan Quan. For the purposes of analysis, only the hue values of the HSV data were used. Black, grey and white data points were omitted from analysis, as they cannot be represented by any unique hue value.

For the rectangular stimuli, the hue values, ranging from 0 to 360 degrees, were grouped into 36 bins of 10 degrees each, and radial histogram plots were generated using Matplotlib (Hunter, 2007). For the radial stimuli, which had a smaller data set, the hue values were grouped into 18 bins of 20 degrees each in plotting the histograms.

The radial histograms display the frequency of colours seen by participants when viewing the stimuli, and allow easy visualisation of the relationship between the colours of the stimuli and the illusory colours that are perceived. 
RESULTS

Factors affecting strength of illusion

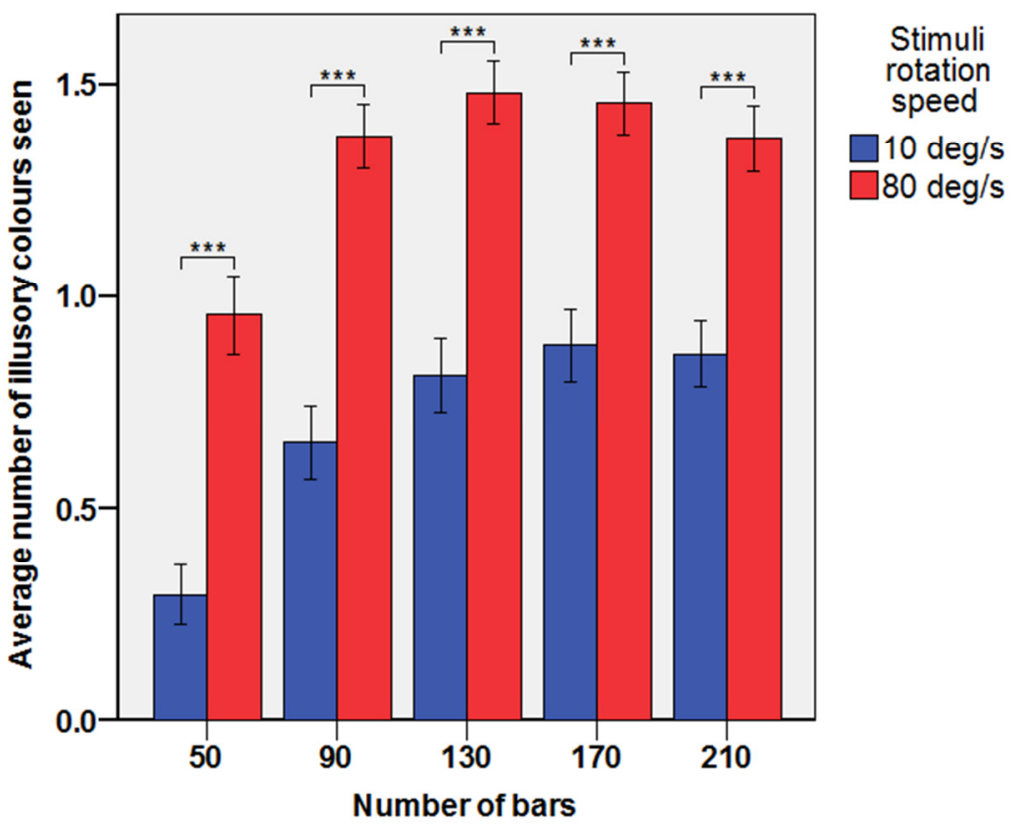

Figure 5. Graph summarising the effects of spatial frequency (number of bars) and speed of stimuli rotation on the strength of the illusion. Vertical lines correspond to \pm 2 standard errors $(n=21)$. ${ }^{* * *} p<0.001$.

For the purposes of analysis, the average number of illusory colours seen by participants is taken to be a proxy of the strength of the illusion. Comparing the average number of illusory colours seen in the 10 conditions (5 spatial frequencies $x$ 2 rotation speeds), the strength of the illusion appears to positively correlate with the spatial frequency and speed of rotation of the stimuli (Figure 5). Further analyses to quantify these relationships are documented below.

\section{Spatial Frequency}

As the number of bars increase from 50 to 170, the average number of illusory colours increases from 0.627 to 1.171 (Figure 6). The strength of the illusion peaks at 170 bars, before decreasing as the number of bars increase beyond 170 bars. Results of the Two-Way Repeated Measures Analysis of Variance (ANOVA) revealed a significant main effect of the spatial frequency, $F(4,84)=54.394, p<$ 0.001 , Cohen's $f=2.14$, on the strength of the illusion. 


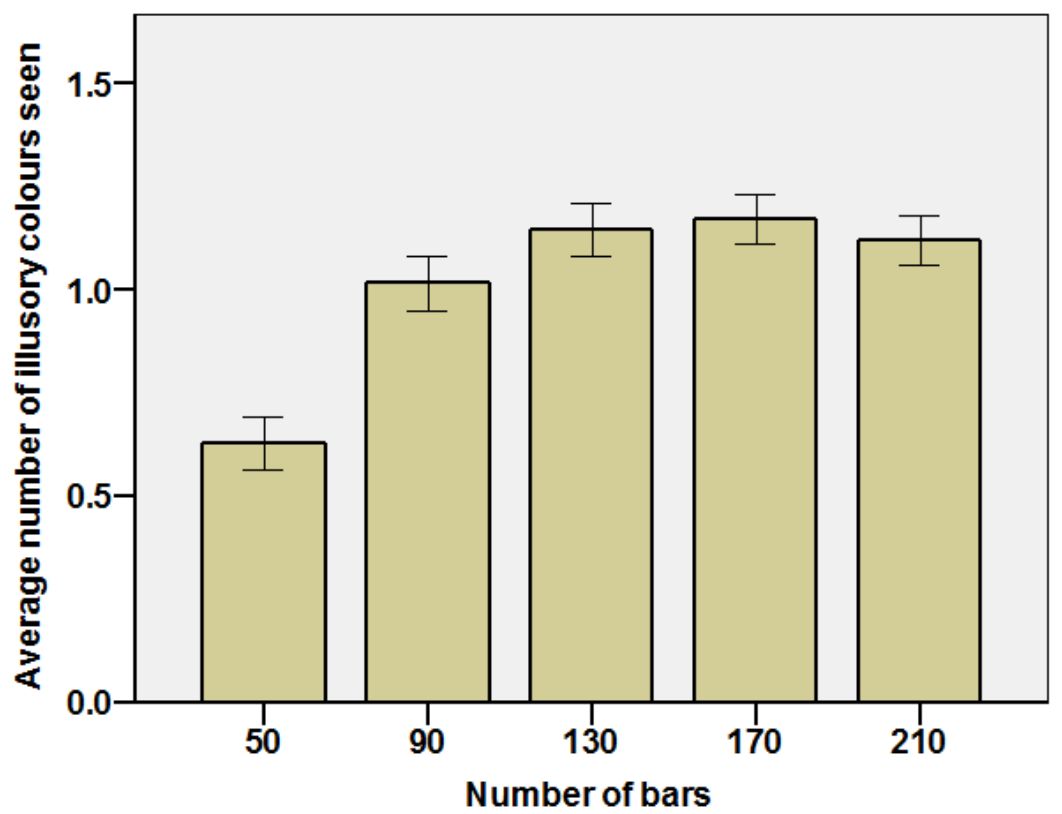

Figure 6. Effect of spatial frequency on strength of illusion. Vertical lines correspond to \pm 2 standard errors $(n=21)$.

\section{Speed of Rotation}

An increase in the speed of rotation from 10 to 80 degrees per second is associated with an increase in the average number of illusory colours seen from 0.703 to 1.329 (Figure 7). Results of the Two-Way Repeated Measures ANOVA reveal a significant main effect of the speed of rotation, $F(1,84)=93.082, p<0.001$, Cohen's $f=2.90$, on the strength of the illusion.

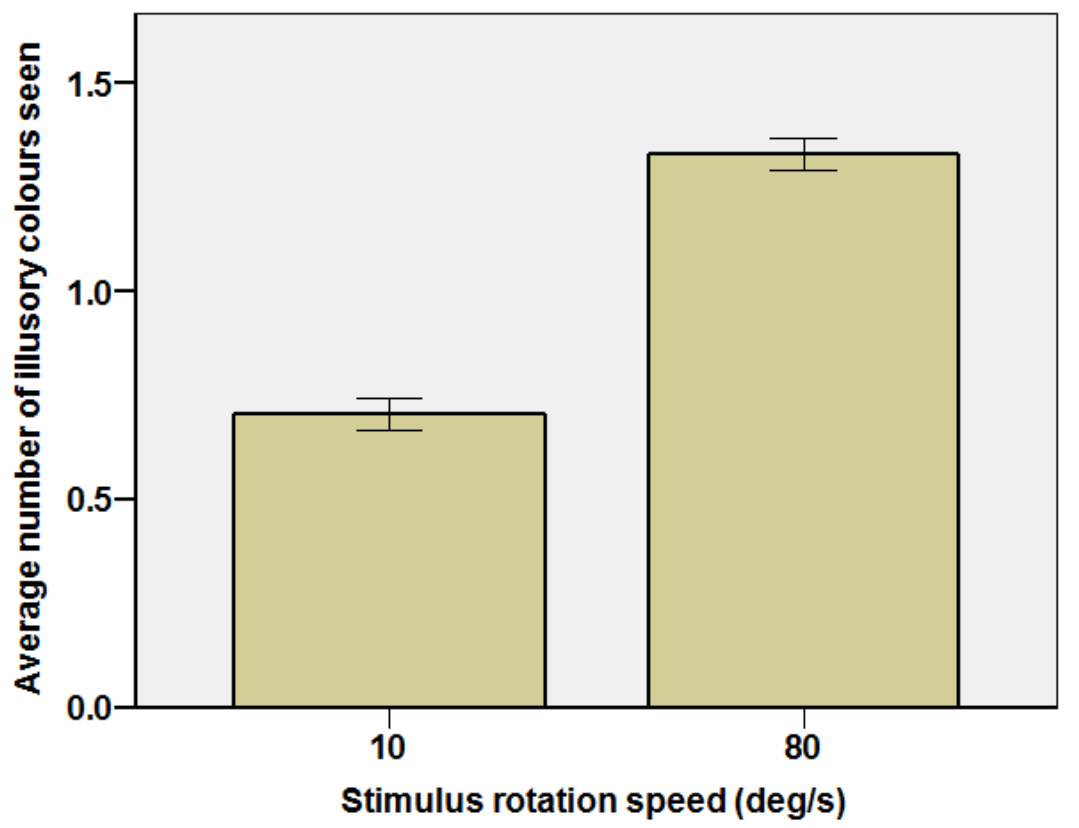

Figure 7. The effect of the rotation speed of the rectangular stimuli on the strength of the illusion. Vertical lines correspond to \pm 2 standard errors $(n=21)$ 
Interaction Effect

A Two-Way Repeated Measures Analysis of Variance was conducted to find out if there was any interaction effect between the spatial frequency and speed of rotation on the strength of the illusion. The results showed that there was a significant interaction effect, $F(4,84)=3.733, p=0.023$, between the two independent variables. Further analysis revealed that although the effect of speed on the strength of the illusion was significant at every spatial frequency condition $(p<0.001)$, the effect of varying the speed was more pronounced at lower spatial frequencies.

\section{Radial Stimuli}

The results of a one-way ANOVA conducted revealed no significant difference $(p=$ 0.929 ) in the average illusion strength when the spatial frequency (number of circles) is varied. As the main aim of employing the static radial stimuli was to study the illusory colours perceived, the range of spatial frequency employed was much smaller, and the lack of variation in illusion strength was expected. 


\section{Illusory colours seen}

Rectangular Stimuli

Rectangular Stimuli - Colours Seen
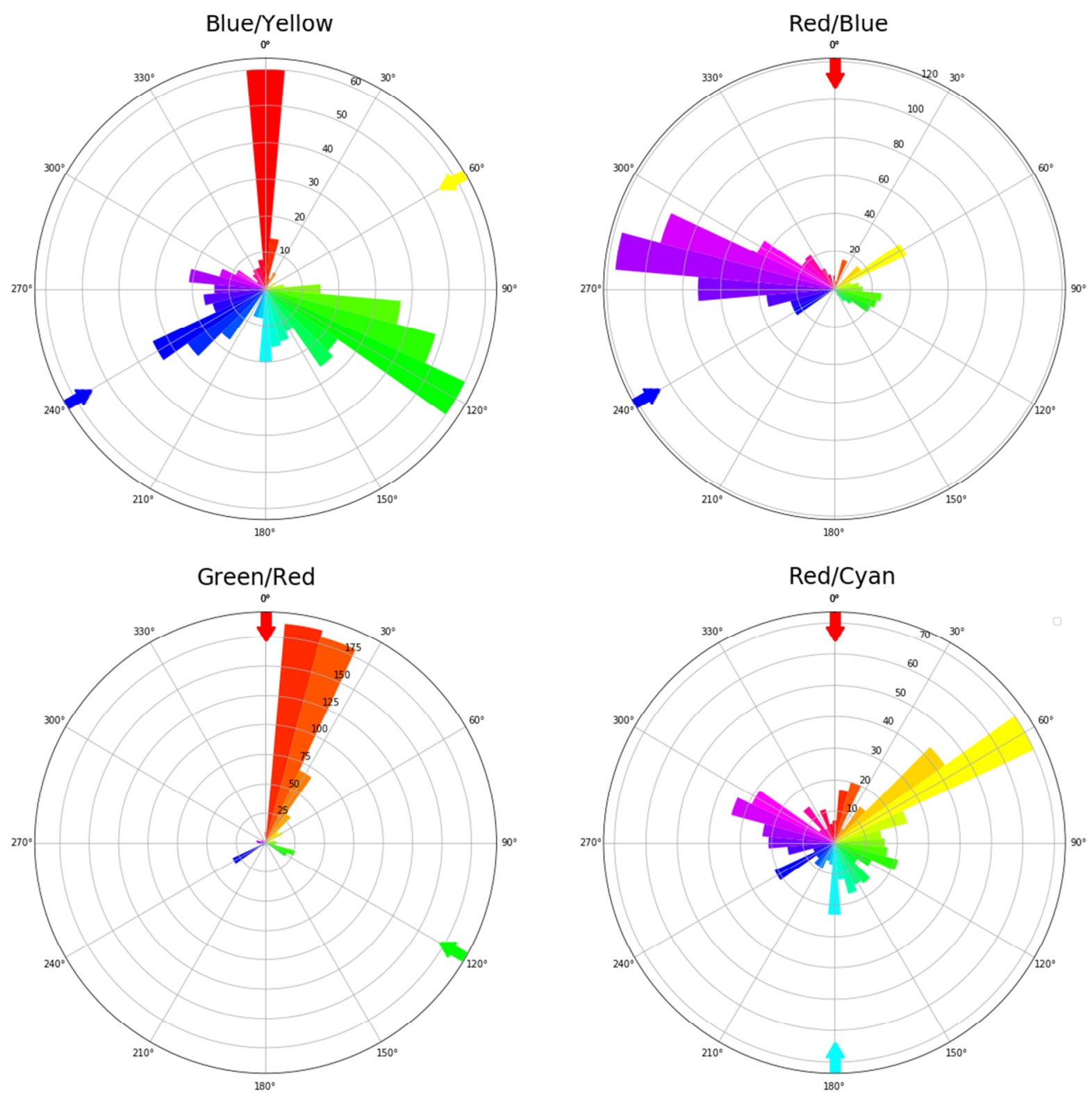

Figure 8. Histograms displaying the frequency of each illusory colour seen in the rotating rectangular stimuli. The radial ticks represent the absolute frequency, while the numbers in degrees represent the corresponding hue value. The coloured arrows indicate the pairs of colours of each group of stimuli.

As shown in Figure 8, the illusory colours seen in the rectangular stimuli comprising blue and yellow bars are bimodal, peaking at red $\left(\mathrm{H}=0^{\circ}, \mathrm{n}=60\right)$ and green $\left(\mathrm{H}=120^{\circ}\right.$, $n=60)$. A smaller peak of blue $\left(H=240^{\circ}, n=34\right)$ is also observed. For the red and blue stimuli, a peak clustering around magenta $\left(\mathrm{H}=280^{\circ}, \mathrm{n}=116\right)$ is observed. For the green and red stimuli, a peak of orange $\left(\mathrm{H}=10^{\circ}, \mathrm{n}=187 ; \mathrm{H}=20^{\circ}, \mathrm{n}=181\right)$ is observed. The red and cyan stimuli, a large peak of yellow $\left(\mathrm{H}=60^{\circ}, \mathrm{n}=70\right)$ and a smaller peak of magenta $\left(\mathrm{H}=290^{\circ}, \mathrm{n}=34\right)$ are observed. 


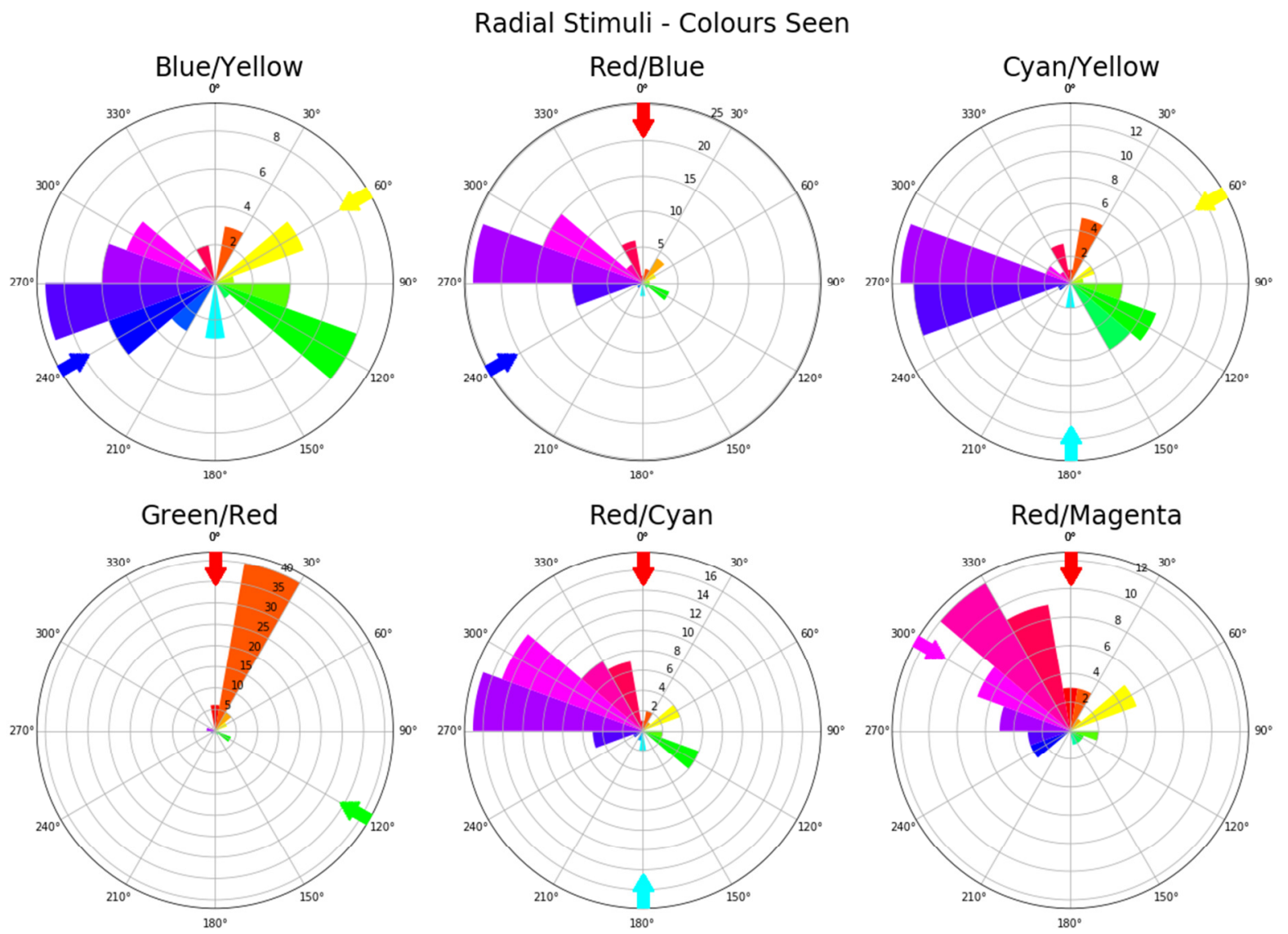

Figure 9. Histograms displaying the frequency of each illusory colour seen in the static radial stimuli. The radial ticks represent the absolute frequency, while the numbers in degrees represent the corresponding hue value. The coloured arrows indicate the pairs of colours of each group of stimuli.

The illusory colours seen in the blue and yellow radial stimuli are clustered around purple $\left(H=260^{\circ}, n=9\right)$, and green $\left(H=120^{\circ}, n=8\right)$. In the red and blue stimuli, one peak at purple $\left(\mathrm{H}=280^{\circ}, \mathrm{n}=24\right)$ is observed. In the cyan and yellow stimuli, a large cluster at purple $\left(H=280^{\circ}, n=13 ; H=260^{\circ}, n=12\right)$ and a smaller peak around green $\left(H=120^{\circ}, n=7 ; H=140^{\circ}, n=6\right)$ is observed. The green and red stimuli had a single large peak $\left(H=20^{\circ}, n=40\right)$, while the red and cyan stimuli had a single clustered peak at purple $\left(H=280^{\circ}, n=17 ; H=300^{\circ}, n=16\right)$. The red and magenta stimuli had a single peak clustered around magenta $\left(H=320^{\circ}, n=12\right)$. 


\section{DISCUSSION}

\section{Factors affecting the strength of the illusion}

\section{Spatial frequency}

The results of the two-way repeated measures ANOVA are in line with our first hypothesis, that the number of illusory colours perceived (strength of illusion) is related to the spatial frequency of the presented stimulus.

At higher spatial frequencies, for any given degree of retinal motion, there is a higher local rate of change in colour on the retina. This result thus supports the possibility that the illusory percept could be generated in a form of successive colour contrast due to von Kries adaptation leading to aftereffect in the retina.

In Blakeslee and McCourt (1999)'s cortical filtering model, the induction of counterphase luminance gratings are accounted for by the summation of inputs from an array of hypercomplex cells in V1, each having a receptive field size corresponding to a particular spatial frequency. The difference in the relative abundance of neurons preferring particular spatial frequencies in V1 or their strength of activation (Mazer et al., 2002) may be responsible for the relationship observed.

\section{Speed of rotation}

The hypothesis that the illusory percept is motion-dependent was derived from initial observations that the percept is extinguished when fixating at the stationary stimulus. The results of this study reveal a significant main effect of speed of rotation on the strength of the illusion ( $p<0.001$ ), affirming the notion that the illusory percept is motion-dependent. This suggests that simultaneous colour contrast cannot be used to explain the illusory percept. Rather, mechanisms of successive colour contrast and von Kries adaptation are implicated.

Due to the different patterns between the rectangular and radial stimuli, direct comparisons of the illusion strength cannot be made. However, it is worthy to note that the average number of illusory colours seen in the radial stimuli $(0.766)$ is higher than that seen in the slow motion condition (0.703), despite the radial stimuli being displayed statically. While this seems to contradict the understanding that motion underlies the illusion, participants were free to move their eyes during the experiment, and the movement generated by their saccades could generate the retinal motion necessary to allow the illusory aftereffects to surface. 


\section{Illusory colours seen}

\section{Non-Complementary Colour Pairs}

In stimuli where non-complementary colour pairs were used (i.e. not 180 hue degrees apart), the hue intermediate between the two colours was consistently produced. This can be attributed to the temporal summation of the two colours as they presented on the same portion of the visual field in rapid succession due to the motion of the stimuli or self-driven eye movements. A similar result was found in a study by Nishida et al. (2007), whereby rapid presentation of alternating red and green stimuli over a motion trajectory produced the perception of a yellow stimulus. In their work, Nishida and colleagues suggested that neurons exhibiting dualselectivity to both motion direction and colour in V1 and V2 could be responsible (Tamura et al., 1996).

In this study, the red-green pair stimuli seemed to produce an illusory intermediate hue closer to red than the expected yellow. This is attributed to the Bezolde-Brucke hue shift, and discussed in the subsequent section.

In the initial study by the team which discovered the illusion (2017), apart from the illusory intermediate hue being perceived, the hue complementary to this intermediate was also frequently observed. For example, when presented with the red-green rectangular stimuli, participants reported seeing yellow, as well as blue. We posited the role of an aftereffect driven by fatigue and von Kries adaptation in explaining in the complementary hue. In this study, however, this phenomenon is only observed in a few of the radial stimuli, and to a much smaller extent. We propose that the low luminance of presentation $\left(10 \mathrm{~cd} / \mathrm{m}^{2}\right)$ facilitated the generation of aftereffects of an even lower intensity, below the salience threshold required for perception and identification.

\section{Complementary Colour Pairs}

In stimuli where complementary colour pairs were used (Blue and Yellow; Red and Cyan), the colour responses tend to be more varied, with two or three main clusters of colours observed. Even so, the results in this group of stimuli challenge our working model of explaining the illusory percept, and present themselves as a novel phenomenon in the field of colour vision science.

Complementary colour pairs, being 180 hue degrees apart, are the colours opposite each other on the hue colour wheel. According to foundational colour theory, 
simultaneous presentation of complementary coloured lights results in the perception of white (achromaticity). In line with Hering's work, any aftereffect generated in response to the illusory white percept, being restricted to the black-white luminance channel, would also be expected to be achromatic.

However, instead of achromatic illusory percepts, the Blue and Yellow stimulus resulted in the illusory perception of red and green, while the Red and Cyan stimulus resulted in an illusory perception of yellow. This illusory percept thus goes beyond the present scope of understanding in the field of colour vision.

In the visual pathway, the three opponent channels are carried by three different fibres - the parvocellular, magnocellular and koniocellular layers - which transmit information from the LGN to V1 (Viqueira Pérez, de Fez, \& Martínez-Verdú, 2010). These channels operate independently, and the effects of adaptation on colour perception have only been documented to occur within the confines of each of the three opponent-colour channels. As the illusory percept appears to cross the independent channels, we posit that this illusion is generated in higher visual areas such as V4 and V5, which are responsible for colour and motion perception respectively, and possible interaction between the two regions.

\section{Limitations}

Comparing the results in Figures 8 and 9, it is apparent that there is a smaller pool of colour response data in the radial set. This is a result of two factors: Firstly, there were significantly fewer trials $(n=45)$ per participant compared to the rectangular stimuli ( $n=120)$. Secondly, on average, participants perceived fewer illusory colours when looking at the radial stimuli. As a result, despite re-grouping the data together in wider bins of 20 hue degrees, the frequencies of each colour reflected in the histograms remain fairly small, and susceptible to the effects of noise. Taking a conservative stance, we abstain from drawing conclusions using this set of data.

Due to the low maximum intensity of the blue phosphor in the CRT used $\left(10 \mathrm{~cd} / \mathrm{m}^{2}\right)$, colours generated using the red and green phosphors had to be scaled down to an equally low intensity in order to achieve stimulus isoluminance. However, studies have shown that colour perception is also governed by the intensity of presentation, a phenomenon known as Bezolde-Brucke hue shift (Pridmore, 1999). At low light intensities, perceived hues shift toward red and green, shifting towards blue and 
yellow at higher intensities. At the low intensities used in this study, the real and illusory hues perceived may have been red- or green-shifted.

Further, while luminance calibration was conducted using a spectrophotometer, absolute isoluminance does not equate to perceptual isoluminance, due to the differences in sensitivities of L, M and S-cones. Thus, although the stimuli are all presented at the same luminance, there may be residual perceptual differences in luminance that affect the hues (real and illusory) perceived (Walraven, 1976).

\section{Future directions}

As this study reveals a new phenomenon going beyond the present scope of knowledge in the field of colour vision, it is of value to seek out the part(s) of the visual pathway responsible for its perception.

In this study, none of the participants had deficiencies in their red-green or blueyellow colour vision (i.e. all were trichromats). In order to elicit the role of photoreceptors and different photoreceptor sensitivities between individuals, future studies involving the illusion could attempt to obtain responses from colour-blind individuals (dichromats) or tetrachromats.

To distinguish between generation of the illusory percept prior to or after the optic chiasm, where information from the two eyes is integrated, future work can present the coloured gratings dichoptically - one colour to each eye. If the illusory percept is extinguished, that would point towards the illusory percept being generated cortically, rather than in the retina.

In the cortex, V1, V2 and V4 regions contain neurons which are sensitive to a particular range of wavelengths, and fire when stimuli of their preferred wavelengths are projected on the retina. Changes in their firing pattern can be recorded via single-cell recording techniques. The grating stimulus documented in this study can be presented to anesthetised monkeys, to see where activation of neurons tuned to the illusory colours perceived begins. For example, the researchers may present the blue-yellow grating on the retina, and look for activation of the red- and greensensitive neurons in each of the colour-sensitive regions of the cortex. 


\section{CONCLUSION}

The perception of colour is governed by the transduction and integration of neural signals from the photoreceptors, through the visual pathway and into the visual cortex. In this study, we presented a novel illusory colour percept generated by isoluminant bi-coloured square-wave gratings. In an exploratory effort, we sought to characterise the illusory percept and the factors that strengthened or weakened its perception. By employing a within-subject repeated measures design, we demonstrated that the strength of illusion is dependent on the spatial frequency and speed of motion. Initial analysis of the results supported our hypotheses, and pointed towards motion aftereffects in the retina as playing a part in generating the percept. We studied the illusory colours elicited by each combination of colours, presenting a novel phenomenon of cross-channel integration between the red-green and blueyellow chrominance channels in the visual pathway. The existence of the illusory percept reveals a gap in the field of colour vision science, with the present theories being insufficient to explain its perception. The study initiated the process of discovering the mechanism underlying the illusory percept and laid the groundwork for future research employing this square-wave grating stimulus, providing a direction for the expansion of colour vision science. 


\section{REFERENCES}

Abney, W. d. W. (1909). On the Change in Hue of Spectrum Colours by Dilution with White Light. Proceedings of the Royal Society of London. Series A, Containing Papers of a Mathematical and Physical Character, 83(560), 120-127.

Blakeslee, B., \& McCourt, M. E. (1999). A multiscale spatial filtering account of the White effect, simultaneous brightness contrast and grating induction. Vision Research, 39(26), 4361-4377. doi:https://doi.org/10.1016/S0042-6989(99)00119-4

Boynton, R. M. (1986). A system of photometry and colorimetry based on cone excitations. Color Research \& Application, 11(4), 244-252.

doi:10.1002/col.5080110405

De Valois, R. L., Smith, C. J., Kitai, S. T., \& Karoly, A. J. (1958). Response of Single Cells in Monkey Lateral Geniculate Nucleus to Monochromatic Light. Science, 127(3292), 238. doi:10.1126/science.127.3292.238

Dvorine, I. (1953). Dvorine Pseudo-Isochromatic Plates (2nd ed.): Harcourt, Brace \& World.

Ejima, Y., \& Takahashi, S. (1984). Bezold-Brücke hue shift and nonlinearity in opponent-color process (Vol. 24).

Ekroll, V. (2005). On the nature of simultaneous colour contrast. Philosophische Fakult"at der Christian-Albrechts-Universit"at zu Kiel.

Gyoba, J. (1983). Stationary phantoms: A completion effect without motion and flicker. Vision Research, 23(2), 205-211. doi:https://doi.org/10.1016/00426989(83)90144-X

Hunter, J. D. (2007). Matplotlib: A 2D Graphics Environment. Computing in Science \& Engineering, 9(3), 90-95. doi:10.1109/MCSE.2007.55

Lu, Z. L., Lesmes, L. A., \& Sperling, G. (1999). The mechanism of isoluminant chromatic motion perception. Proceedings of the National Academy of Sciences of the United States of America, 96(14), 8289-8294.

Mazer, J. A., Vinje, W. E., McDermott, J., Schiller, P. H., \& Gallant, J. L. (2002). Spatial frequency and orientation tuning dynamics in area V1. Proceedings of the National Academy of Sciences of the United States of America, 99(3), 1645-1650. doi:10.1073/pnas.022638499

McCourt, M. (1994). Grating induction: a new explanation for stationary phantom gratings. Vision Research, 34(12), 1609-1617. doi:https://doi.org/10.1016/00426989(94)90118-X

Nishida, S. y., Watanabe, J., Kuriki, I., \& Tokimoto, T. (2007). Human Visual System Integrates Color Signals along a Motion Trajectory. Current Biology, 17(4), 366-372. doi:https://doi.org/10.1016/j.cub.2006.12.041

Peirce, J., Gray, J. R., Simpson, S., MacAskill, M., Höchenberger, R., Sogo, H., . . . Lindeløv, J. K. (2019). PsychoPy2: Experiments in behavior made easy. Behavior Research Methods, 51(1), 195-203. doi:10.3758/s13428-018-01193-y

Pridmore, R. W. (1999). Bezold-Brucke hue-shift as functions of luminance level, luminance ratio, interstimulus interval and adapting white for aperture and object 
colors. Vision Research, 39(23), 3873-3891. doi:https://doi.org/10.1016/S00426989(99)00085-1

Pridmore, R. W. (2013). Single cell spectrally opposed responses: opponent colours or complementary colours? Journal of Optics, 42(1), 8-18. doi:10.1007/s12596-0120090-0Svaetichin, G., \& MacNichol Jr, E. F. (1958). Retinal Mechanisms For Chromatic And Achromatic Vision. Annals of the New York Academy of Sciences, 74(2), 385-404. doi:10.1111/j.1749-6632.1958.tb39560.x

Troscianko, T., Montagnon, R., Le Clerc, J., Malbert, E., \& L Chanteau, P. (1991). The Role of Colour as a Monocular Depth Cue (Vol. 31).

Viqueira Pérez, V., de Fez, D., \& Martínez-Verdú, F. (2010). Colour vision: Theories and principles (pp. 3-e2).

Walraven, J. (1976). Discounting the background - the missing link in the explanation of chromatic induction. Vision Research, 16(3), 289-295. doi:https://doi.org/10.1016/0042-6989(76)90112-7

White, M. (1979). A New Effect of Pattern on Perceived Lightness. Perception, 8(4), 413-416. doi:10.1068/p080413

Whittle, P., \& Challands, P. D. C. (1969). The effect of background luminance on the brightness of flashes. Vision Research, 9(9), 1095-1110.

doi:https://doi.org/10.1016/0042-6989(69)90050-9

Wiesel, T. N., \& Hubel, D. H. (1966). Spatial and chromatic interactions in the lateral geniculate body of the rhesus monkey. Journal of Neurophysiology, 29(6), 11151156. doi:10.1152/jn.1966.29.6.1115

Zeki, S. (1983). Colour coding in the cerebral cortex: The reaction of cells in monkey visual cortex to wavelengths and colours. Neuroscience, 9(4), 741-765.

doi:https://doi.org/10.1016/0306-4522(83)90265-8 